\title{
Drift Waves, Intense Parallel Electric Fields, And Turbulence Associated With Asymmetric Magnetic Reconnection At The Magnetopause
}
R. E. Ergun
L.-J. Chen
F. D. Wilder
P. A. Cassak

Follow this and additional works at: https://researchrepository.wvu.edu/faculty_publications

\section{Digital Commons Citation}

Ergun, R. E.; Chen, L.-J.; Wilder, F. D.; and Cassak, P. A., "Drift Waves, Intense Parallel Electric Fields, And Turbulence Associated With Asymmetric Magnetic Reconnection At The Magnetopause" (2017). Faculty \& Staff Scholarship. 957.

https://researchrepository.wvu.edu/faculty_publications/957

This Article is brought to you for free and open access by The Research Repository @ WVU. It has been accepted for inclusion in Faculty \& Staff Scholarship by an authorized administrator of The Research Repository @ WVU. For more information, please contact beau.smith@mail.wvu.edu. 


\section{Geophysical Research Letters}

\section{RESEARCH LETTER}

10.1002/2016GL072493

\section{Key Points:}

- Asymmetric magnetic reconnection at the magnetopause often has strong magnetic field fluctuations and intense parallel electric fields

- The magnetic field fluctuations are consistent with a thin, oscillating current sheet that is corrugated along the $\mathrm{X}$ line (drift wave)

- The drift waves appear to drive strong parallel currents which, in turn, generate large-amplitude parallel electric fields

\section{Correspondence to:}

R. E. Ergun,

ree@lasp.colorado.edu

\section{Citation:}

Ergun, R. E., et al. (2017), Drift waves, intense parallel electric fields, and turbulence associated with asymmetric magnetic reconnection at the magnetopause, Geophys. Res. Lett., 44, 2978-2986, doi:10.1002/2016GL072493.

Received 30 DEC 2016 Accepted 21 MAR 2017

Accepted article online 23 MAR 2017 Published online 9 APR 2017

\section{Drift waves, intense parallel electric fields, and turbulence associated with asymmetric magnetic reconnection at the magnetopause}

\author{
R. E. Ergun 1,2 (D) L.-J. Chen ${ }^{3,4}$ (D, F. D. Wilder ${ }^{2}$ (D, N. Ahmadi ${ }^{2}$ (D) S. Eriksson ${ }^{2}$ (D, M. E. Usanova² (D), \\ K. A. Goodrich ${ }^{1,2}$ (D) J. C. Holmes ${ }^{1,2}$ (D) A. P. Sturner ${ }^{1,2}$ (D) D. M. Malaspina ${ }^{2}$ D, D. L. Newman ${ }^{2,5}$ (D), \\ R. B. Torbert ${ }^{6,7}$ D, M. R. Argall ${ }^{6}$ (D, P.-A. Lindqvist $^{8}$ (D, J. L. Burch ${ }^{7}$ D, J. M. Webster ${ }^{7}$, J. F. Drake ${ }^{3}$ (D), \\ L. Price ${ }^{3}$ iD, P. A. Cassak ${ }^{\text {ID }}$, M. Swisdak ${ }^{3}$ D, M. A. Shay ${ }^{10}$ iD, D. B. Graham ${ }^{11}$ (D, R. J. Strangeway ${ }^{12}$ (D), \\ C. T. Russell ${ }^{12}$ (D) B. L. Giles ${ }^{3}$ (D) J. C. Dorelli ${ }^{3}$, D. Gershman ${ }^{3,4}$ (D) L. Avanov ${ }^{3}$ (D) M. Hesse ${ }^{3}$,

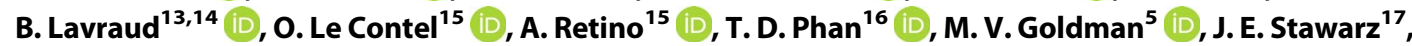 \\ S. J. Schwartz ${ }^{17}$ D, J. P. Eastwood ${ }^{17}$ iD, K.-J. Hwang ${ }^{3}$, R. Nakamura ${ }^{18}$ iD, \\ and S. Wang ${ }^{3,4}$ (D)
}

${ }^{1}$ Department of Astrophysical and Planetary Sciences, University of Colorado, Boulder, Colorado, USA, ${ }^{2}$ Laboratory of Atmospheric and Space Sciences, University of Colorado, Boulder, Colorado, USA, ${ }^{3}$ NASA, Goddard Space Flight Center, Greenbelt, Maryland, USA, ${ }^{4}$ IREAP, University of Maryland, College Park, Maryland, USA, ${ }^{5}$ Department of Physics, University of Colorado, Boulder, Colorado, USA, ${ }^{6}$ University of New Hampshire, Durham, New Hampshire, USA, ${ }^{7}$ Southwest Research Institute, San Antonio, Texas, USA, ${ }^{8}$ Royal Institute of Technology, Stockholm, Sweden, ${ }^{9}$ West Virginia University, Morgantown, West Virginia, USA, ${ }^{10}$ University of Delaware, Newark, Delaware, USA, ${ }^{11}$ Swedish Institute of Space Physics, Uppsala, Sweden, ${ }^{12}$ University of California, Los Angeles, Los Angeles, California, USA, ${ }^{13}$ Institut de Recherche en Astrophysique et Planétologie, Université de Toulouse, Toulouse, France, ${ }^{14}$ Centre National de la Recherche Scientifique, Toulouse, France, ${ }^{15}$ Laboratoire de Physique des Plasmas, Palaiseau, France, ${ }^{16}$ Space Sciences Laboratory, University of California, Berkeley, California, USA, ${ }^{17}$ The Blackett Laboratory, Imperial College London, London, United Kingdom, ${ }^{18}$ Space Research Institute, Austrian Academy of Sciences, Graz, Austria

Abstract Observations of magnetic reconnection at Earth's magnetopause often display asymmetric structures that are accompanied by strong magnetic field $(\boldsymbol{B})$ fluctuations and large-amplitude parallel electric fields $\left(E_{\|}\right)$. The $\boldsymbol{B}$ turbulence is most intense at frequencies above the ion cyclotron frequency and below the lower hybrid frequency. The $\boldsymbol{B}$ fluctuations are consistent with a thin, oscillating current sheet that is corrugated along the electron flow direction (along the $X$ line), which is a type of electromagnetic drift wave. Near the $X$ line, electron flow is primarily due to a Hall electric field, which diverts ion flow in asymmetric reconnection and accompanies the instability. Importantly, the drift waves appear to drive strong parallel currents which, in turn, generate large-amplitude $(\sim 100 \mathrm{mV} / \mathrm{m}) E_{\|}$in the form of nonlinear waves and structures. These observations suggest that turbulence may be common in asymmetric reconnection, penetrate into the electron diffusion region, and possibly influence the magnetic reconnection process.

\section{Introduction}

Magnetic reconnection at the magnetopause often has asymmetric structure [e.g., Cassak and Shay, 2007] due to a significant difference between the properties of the plasma in the magnetosheath and the properties of the plasma in the magnetosphere [e.g., Paschmann et al., 2013 and references therein]. Magnetosheath plasmas have "warm" electron and ion temperatures $\left(T_{\mathrm{e}} \sim 100 \mathrm{eV}\right.$ and $\left.T_{\mathrm{i}} \sim 400 \mathrm{eV}\right)$ and densities $(n)$ of tens of $\mathrm{cm}^{-3}$. In contrast, the plasma in the magnetosphere has significantly lower densities $\left(\sim 1 \mathrm{~cm}^{-3}\right)$ but higher temperatures. Often, $T_{\mathrm{e}}$ is $\sim 400 \mathrm{eV}$ and $T_{\mathrm{i}}$ is $\sim 1 \mathrm{keV}$ or greater. Interestingly, the magnetosphere may contain a cold (<10 eV) plasma component as well [Su et al., 2000; McFadden et al., 2008].

Because of its higher density, the inflow to a magnetic reconnection region from the magnetosheath carries a substantial ion momentum flux, which flows past the $X$ line and is ultimately slowed and diverted by a Hall electric that is field normal to the magnetopause (often in the geocentric solar ecliptic (GSE) $X$ direction, $E_{X}$ ) on the magnetosphere side of the $X$ line [e.g., Cassak and Shay, 2007; Hesse et al., 2014, 2016; Burch et al., 2016b]. Typical values of $E_{X}$ are on the order of $10 \mathrm{mV} / \mathrm{m} . E_{X}$ is confined to a thin layer in the $X$ direction with a scale size greater than the electron gyroradius $\left(\rho_{\mathrm{e}}\right)$ but less than the ion gyroradius $\left(\rho_{\mathrm{i}}\right)$. As a result, $E_{X}$ acts directly on ions but produces a strong $\boldsymbol{E} \times \boldsymbol{B}$ electron drift along the magnetic reconnection $\mathbf{X}$ line 
(typically the GSE $-Y$ direction). $\boldsymbol{B}$, the magnetic field, is often in the GSE $Z$ direction on the magnetosphere side of antiparallel reconnection. The electron drift due to $E_{X}$ supplies the current in the $Y$ direction as part of the large-scale magnetopause current sheet. In other words, $E_{X}$, generated by ion ram pressure, drives the current local to the magnetic reconnection $X$ line, replacing a gradient in electron pressure $\left(\nabla P_{\mathrm{e}}\right)$ or ion pressure $\left(\nabla P_{\mathrm{i}}\right)$, which drives the magnetopause current sheet elsewhere.

Magnetospheric Multiscale (MMS) observations [e.g., Burch et al., 2016b; Burch and Phan, 2016; Chen et al., 2016] and simulations [Cassak and Shay, 2007; Hesse et al., 2014, 2016] have demonstrated that $E_{X}$ is a characteristic feature of asymmetric magnetic reconnection. However, MMS observations also show that strong fluctuations in $\boldsymbol{B}$ with frequencies between $\sim 1 \mathrm{~Hz}$ and $\sim 10 \mathrm{~Hz}$ are often present in asymmetric magnetic reconnection [Ergun et al., 2016a]. Similar characteristics also have been reported in 3D simulations of magnetic reconnection [Li et al., 2012; Roytershteyn et al., 2013; Egedal et al., 2015; Lapenta et al., 2015; Price et al., 2016]. In the observations, however, the $\boldsymbol{B}$ fluctuations are often accompanied by large-amplitude $(\sim 100 \mathrm{mV} / \mathrm{m})$, parallel electric fields $\left(E_{\|}\right)$that appear as nonlinear structures [Ergun et al., 2016a]. We note that wave-like $E_{\|}$can result from the mixing of the warm, dense magnetosheath plasma with cold electrons in the magnetosphere [Ergun et al., 2016b], which we do not address in this article.

In this article, we show that the thin current sheets that are driven by $E_{x}$ appear to be unstable to a type of electromagnetic drift wave. A "textbook" drift wave [e.g., Chen, 1984] travels at the electron drift speed assumed to be driven by an electron pressure gradient $\left(\nabla P_{\mathrm{e}}\right)$ and has a finite wave vector $(\boldsymbol{k})$ component along $\boldsymbol{B}$. The observed drift waves are similar to classic drift waves in that they travel at the electron drift speed and appear to have finite $k_{\|}$. The electron motion, however, is dominated by the Hall electric field, $E_{x}$, rather than $\nabla P_{\mathrm{e}}$.

The energy source exciting the observed drift waves is difficult to unequivocally identify. Ion ram pressure appears to generate the strong $E_{X}$ and, hence, strong current. One possibility is a modified twostream instability [McBride et al., 1972; Ji et al., 2004, 2005] from the strong electron drift or from ion motion [Wang et al., 2016]. The observed waves also have some characteristics of a lower hybrid drift instability (LHDI) [Krall and Liewer, 1971; Yoon et al., 2008], which has been suggested to occur in the magnetopause region [Bale et al., 2002; Daughton, 2003; Zhou et al., 2009; Pritchett et al., 2012]. However, the observed waves are not entirely consistent with the classic derivation of the LHDI, which we discuss later.

We examine six events confirmed as asymmetric magnetic reconnection [Burch et al., 2016b; Chen et al., 2016; Burch and Phan, 2016; Phan et al., 2016], five of which display significant $\boldsymbol{B}$ and $E_{\|}$fluctuations. In one event, the MMS spacecraft did not fully enter the magnetopause, so we are unable to determine if there were significant $\boldsymbol{B}$ and $E_{\|}$fluctuations. Even with such small statistics, these observations suggest that asymmetric magnetic reconnection may be inherently turbulent. Furthermore, the low-frequency $\boldsymbol{B}$ fluctuations and the strong $E_{\|}$fluctuations seem to be connected through strong field-aligned currents [Roux et al., 2011; Stawarz et al., 2015]. The low-frequency $\boldsymbol{B}$ fluctuations and the strong $E_{\|}$fluctuations may influence magnetic reconnection.

\section{Observations}

The MMS mission [Burch et al., 2016a] is designed to study magnetic reconnection at high temporal and spatial resolution. The first phase of the mission studies the magnetopause. This region has been observed at lower resolution [Paschmann et al., 2013] and studied with numerical simulations and analytic analysis [e.g., Shay et al., 1998; Hesse and Winske, 1998; Birn et al., 2001; Drake et al., 2006; Lapenta et al., 2006; Cassak and Shay, 2007; Burch and Drake, 2009].

Figure 1 displays two examples of magnetic reconnection observed by MMS that have been previously published. Figure 1a displays $5 \mathrm{~s}$ of $\boldsymbol{B}$ [Torbert et al., 2016; Russell et al., 2016] in GSE coordinates; the colors represent the directions. The black trace is $|\boldsymbol{B}|$. The MMS spacecraft are near an electron diffusion region (EDR) of magnetic reconnection [Burch et al., 2016b]. The EDR encounter was just after 13:07:02 UT. We call attention to the region between the two vertical dashed lines prior to the EDR encounter, which is in the magnetopause current sheet. All components of $\boldsymbol{B}$ and $|\boldsymbol{B}|$ have strong, visible fluctuations in the $1 \mathrm{~Hz}$ to $10 \mathrm{~Hz}$ 

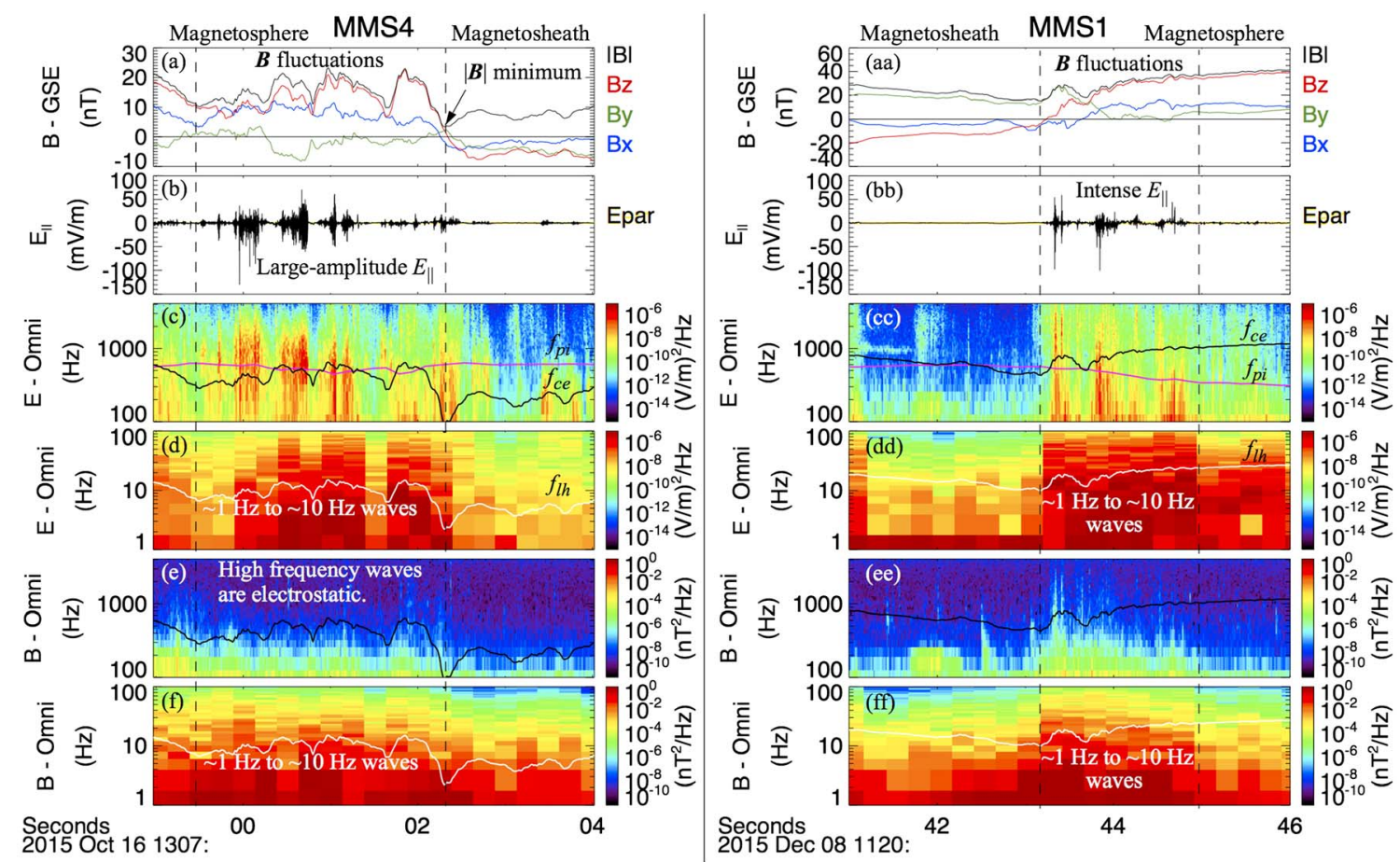

Figure 1. (left) MMS 4 observations on 16 October 2015 of a magnetic reconnection region [Burch et al., 2016b]. (a) B in GSE coordinates. B fluctuations are discernable between the vertical dashed lines. (b) $E_{\|}$at 8196 samples/s. Intense $E_{\|}$fluctuations are concurrent with the $\boldsymbol{B}$ fluctuations. (c and d) $\boldsymbol{E}$ spectral power density as a function of frequency. The magenta trace is $f_{\mathrm{pi}}$, the black trace is $f_{\mathrm{ce}}$, and the white line is $f_{\mathrm{lh}}$. (e and $f$ ) $\boldsymbol{B}$ spectral power density as a function of frequency. The most intense wave power is below the lower hybrid frequency. The ion cyclotron frequency (not shown) is below $1 \mathrm{~Hz}$ at all times. (right) MMS 1 observations on 16 October 2015 of a magnetic reconnection region [Burch and Phan, 2016]. The data show similar characteristics as seen on the left panel. $\boldsymbol{B}$ fluctuations are concurrent with intense $E_{\|}$. The most powerful $\boldsymbol{B}$ fluctuations are in the $1 \mathrm{~Hz}$ to $10 \mathrm{~Hz}$ band.

frequency range. At the same time, $E_{\|}$[Torbert et al., 2016; Lindqvist et al., 2016, Ergun et al., 2016c] displays $\sim 100 \mathrm{mV} / \mathrm{m}$ fluctuations at much higher frequencies (Figure 1b). The $E_{\|}$signal is a combination of nonlinear waves and nonlinear structures [Ergun et al., 2016a]. Figures 1c-1f display the omnidirectional spectral power density of $\boldsymbol{E}$ and $\boldsymbol{B}$. The $\boldsymbol{B}$ spectra are from search coil magnetometer signals [Le Contel et al., 2016]. The high-frequency $\boldsymbol{E}$ power (Figure 1c) corresponds to the bursts of large-amplitude $E_{\|}$ (Figure 1b) as well as perpendicular electric fields (not shown). There is little enhanced power in $\boldsymbol{B}$ at high frequencies (Figure 1e) indicating that the $E_{\|}$fluctuations are primarily electrostatic. However, both $\boldsymbol{E}$ (Figure 1d) and $\boldsymbol{B}$ (Figure 1f) show enhanced emissions in the $\sim 1 \mathrm{~Hz}$ to $\sim 10 \mathrm{~Hz}$ range. This frequency range is higher than the ion cyclotron frequency $\left(f_{\mathrm{ci}}\right.$ is between $0.1 \mathrm{~Hz}$ and $\left.1 \mathrm{~Hz}\right)$ and lower than the lower hybrid frequency $\left(f_{\mathrm{Ih}}\right)$, which is plotted on the spectra as a white trace. Such wave spectra have been reported earlier [Bale et al., 2002; Pritchett et al., 2012].

The right side of Figure 1 plots another published event of an EDR encounter [Burch and Phan, 2016]. The right plot has the same format and period ( $5 \mathrm{~s}$ ) as the left plot. Again, strong $\boldsymbol{B}$ fluctuations are seen on the magnetosphere side current sheet (Figure 1aa). These fluctuations are concurrent with bursts of largeamplitude $E_{\|}$fluctuations (Figure $1 \mathrm{bb}$ ). The high-frequency $E_{\|}$fluctuations are primarily electrostatic (Figures 1cc and 1ee). There is enhanced wave power in the $\sim 1 \mathrm{~Hz}$ to $\sim 10 \mathrm{~Hz}$ band, which is higher than $f_{\mathrm{ci}}$ and lower than $f_{\mathrm{lh}}$.

Similar $\boldsymbol{B}$ fluctuations and $E_{\|}$fluctuations are seen in two other confirmed asymmetric EDR encounters: 19 September 2015 at 09:09:58 UT and 22 October 2015 at 06:05:20 UT [Phan et al., 2016; Ergun et al., 2016b]. During another EDR encounter on 19 September 2015 07:43:30 UT [Chen et al., 2016], the MMS spacecraft did not fully enter the magnetosphere so such fluctuations could not have been detected. With this preliminary survey, it appears that $\boldsymbol{B}$ and $\boldsymbol{E}$ fluctuations from $\sim 1 \mathrm{~Hz}$ to $10 \mathrm{~Hz}$ concurrent with large-amplitude $E_{\|}$fluctuations at higher frequencies $(>\sim 50 \mathrm{~Hz})$ may be characteristic of asymmetric magnetic reconnection at Earth's dayside. 

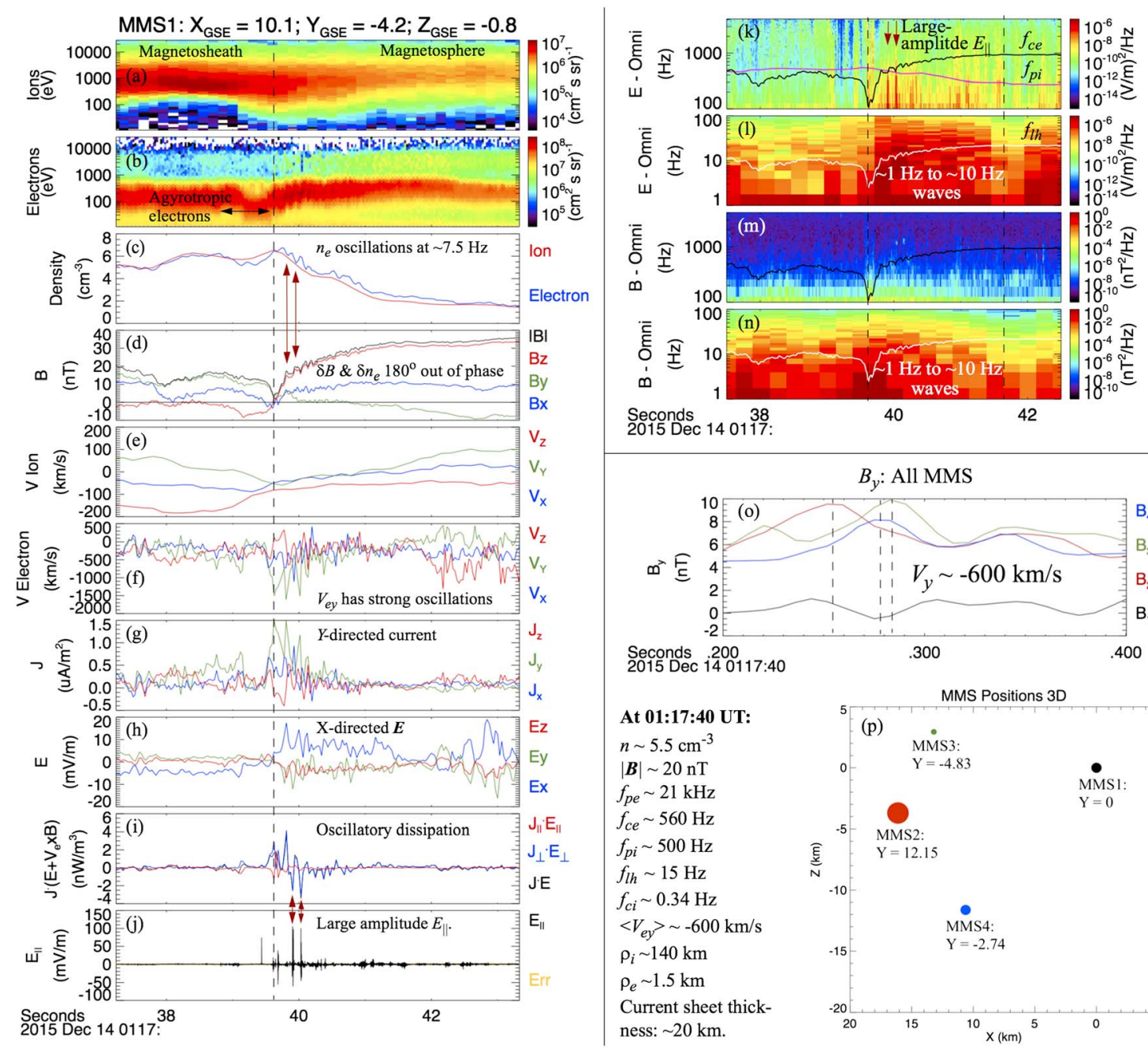

Seconds 140138 Dec 140117

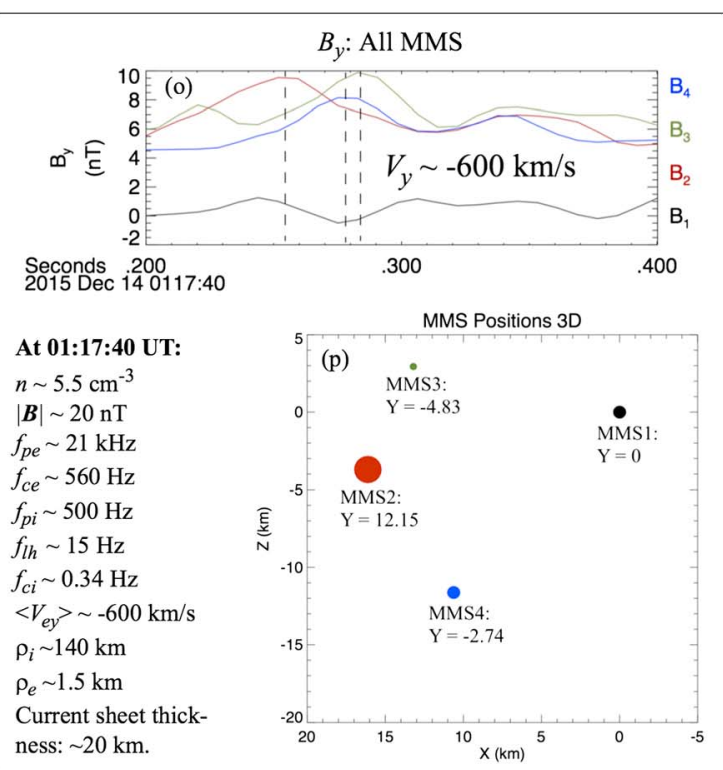

Figure 2. Observations of an EDR encounter. ( $a$ and $b$ ) The differential ion and electron energy fluxes. (c) lon and electron densities. (d) B. (e) lon velocity. ( $f$ ) Electron velocity. (g) Current. (h) Electric field. (i) Energy dissipation. (j) $E_{\|}$at 8196 samples/s. (k and l) The electric field spectral power density. (m and $\mathrm{n}$ ) The magnetic field spectral power density as a function of frequency. The magenta trace is $f_{\mathrm{pi}}$, the black trace is $f_{\mathrm{ce}}$, and the white line is $f_{\mathrm{lh}}$. (0) An expanded view of $B_{y}(0.2 \mathrm{~s})$ from all four MMS spacecraft. (p) The positions of the MMS spacecraft in GSE coordinates. Signal delays indicate that $\boldsymbol{B}$ fluctuations have $V_{y} \sim-600 \mathrm{~km} / \mathrm{s}$.

We examine a sixth asymmetric EDR encounter in detail (Figure 2). Figures $2 \mathrm{a}$ and $2 \mathrm{~b}$ display ion and electron differential energy fluxes (color) as a function of energy (vertical axis) and time [Pollock et al., 2016]. The horizontal axis covers $5 \mathrm{~s}$. The electron and ion fluxes indicate that the spacecraft was in the magnetosheath prior to 01:17:39.6 UT, after which it entered the magnetosphere side of the magnetopause. Figure $2 \mathrm{c}$ plots ion $\left(n_{\mathrm{i}}\right)$ and electron $\left(n_{\mathrm{e}}\right)$ densities [Pollock et al., 2016]. The ion density (red trace) is measured every $150 \mathrm{~ms}$ ( $3.33 \mathrm{~Hz}$ Nyquist frequency) whereas $n_{\mathrm{e}}$ (blue trace) is measured every $30 \mathrm{~ms}(16.7 \mathrm{~Hz}$ Nyquist frequency). Visible oscillations in $n_{\mathrm{e}}$ at $\sim 7.5 \mathrm{~Hz}$ are seen at $\sim$ 01:17:40 UT. The oscillations are not seen in $n_{\mathrm{i}}$ due to the lower sampling rate. Any low-frequency $(<3.3 \mathrm{~Hz})$ differences between the two traces are due to measurement error; the plasma is quasineutral.

Figure $2 \mathrm{~d}$ displays $B$ in GSE coordinates. Fluctuations in the $\sim 1 \mathrm{~Hz}$ to $\sim 10 \mathrm{~Hz}$ frequency range are visible in all three components and in $|\boldsymbol{B}|$ from the beginning of the figure until 01:17:42 UT. The vertical red arrows show that the oscillations in $|\boldsymbol{B}|$ are $\sim 180^{\circ}$ out of phase with $n_{\mathrm{e}}$ oscillations. Figures $2 \mathrm{e}$ and $2 \mathrm{f}$ plot the ion $\left(\boldsymbol{V}_{\mathrm{i}}\right.$, sampled every $150 \mathrm{~ms}$ ) and electron $\left(\boldsymbol{V}_{\mathrm{e}}\right.$, sampled every $30 \mathrm{~ms}$ ) velocities. At 01:17:40 UT, $\boldsymbol{V}_{\mathrm{e}}$ is predominantly in the $-Y$ direction producing a strong current in the $+Y$ direction (Figure $2 \mathrm{~g}$ ). $\boldsymbol{V}_{\mathrm{e}}$ shows strong oscillations in the $\sim 1 \mathrm{~Hz}$ to $\sim 10 \mathrm{~Hz}$ frequency band. $\boldsymbol{V}_{\mathrm{i}}$ is undersampled but could have fluctuations at much lower amplitudes (see discussion below). $\boldsymbol{V}_{\mathrm{e}}$ dominates the current $(\boldsymbol{J})$. 
Figure $2 \mathrm{~h}$ displays $\boldsymbol{E}$ sampled every $31.25 \mathrm{~ms}$ ( $16 \mathrm{~Hz}$ Nyquist). At 01:17:40 UT, $\boldsymbol{E}$ is mostly in the $X$ direction and consistent with $\boldsymbol{V}_{\mathrm{e}}$ such that $\boldsymbol{E}+\boldsymbol{V}_{\mathrm{e}} \times \boldsymbol{B}<\sim 1 \mathrm{mV} / \mathrm{m}$ for most of the region. Visible fluctuations in $\boldsymbol{E}$ are also in the $\sim 1 \mathrm{~Hz}$ to $\sim 10 \mathrm{~Hz}$ frequency range. Figure $2 \mathrm{i}$ displays dissipation in the electron frame, $\langle\boldsymbol{J}\rangle \cdot\left(\langle\boldsymbol{E}\rangle+\left\langle\boldsymbol{V}_{\mathrm{e}}\right\rangle \times\langle\boldsymbol{B}\rangle\right)$, where the brackets indicate an averaging over $\sim 30 \mathrm{~ms}$. Figure $2 \mathrm{i}$ does not include possible dissipation from the large-amplitude, high-frequency waves and nonlinear structures in Figure 2j. In Figure 2j, $E_{\|}$is sampled 8196 times per second. Interestingly, the two negative peaks in dissipation, marked by red arrows between Figure $2 \mathrm{i}$ and Figure $2 \mathrm{j}$, are in the perpendicular components and coincide with strong peaks in $E_{\|}$. These data suggest that the low-frequency $(\sim 1 \mathrm{~Hz}$ to $\sim 10 \mathrm{~Hz})$ waves may be supplying the energy for the strong $E_{\|}$.

\section{Analysis and Model of the Observed Waves}

Figure 2 shows that the low-frequency fluctuations seen in $\boldsymbol{B}$ are also seen in $n_{\mathrm{e}}, \boldsymbol{E}, \boldsymbol{V}_{\mathrm{e}}$, and $\boldsymbol{J}$. The visible oscillations are at $\sim 7.5 \mathrm{~Hz}$, as measured from the period between peaks in $\boldsymbol{B}$ and $n_{\mathrm{e}}$. As in Figure 1, Figures $2 \mathrm{k}$ and $2 \mathrm{l}$, which display the spectral power density of $\boldsymbol{E}$, and Figures $2 \mathrm{~m}$ and $2 \mathrm{n}$, which display the spectral power density of $\boldsymbol{B}$, indicate that the majority of the low-frequency power is in the $\sim 1 \mathrm{~Hz}$ to $\sim 10 \mathrm{~Hz}$ range. This frequency range is higher than $f_{\mathrm{ci}}$ but lower than $f_{\mathrm{lh}}$. A timing analysis using multiple MMS spacecraft (Figures 20 and $2 p$ ) indicates that the low-frequency fluctuations in $\boldsymbol{B}$ are traveling primarily in the $-Y$ direction at $600 \mathrm{~km} / \mathrm{s}+300 \mathrm{~km} / \mathrm{s}$.

Ignoring the fluctuations, the data indicate a current sheet crossing near the EDR. At 01:17:39.6, $B_{z}$ rises rapidly consistent with current in the $Y$ direction. The change in $B_{z}(\sim 40 \mathrm{nT})$ and the peak values of $J_{y}$ $\left(1.5 \mu \mathrm{A} / \mathrm{m}^{2}\right)$ imply a current sheet thickness of $\sim 20 \mathrm{~km}$, which is less than the ion gyroradius $\left(\rho_{\mathrm{i}} \sim 140 \mathrm{~km}\right.$ at 01:17:40 UT) but larger than the electron gyroradius $\left(\rho_{\mathrm{e}} \sim 1.5 \mathrm{~km}\right.$ at 01:17:40 UT) and electron skin depth $\left(\delta_{\mathrm{e}} \sim 2 \mathrm{~km}\right)$. This current layer thickness near the EDR $\left(\sim 10 \delta_{\mathrm{e}}\right)$ compares well to that seen in laboratory experiments [Ji et al., 2008]. $J_{y}$ is driven primarily by the Hall electric field $\left(E_{x}\right)$ after 01:17:40 UT. $\mid \nabla P_{\mathrm{e}} /$ en $_{\mathrm{e}} \mid$ (see later) is less than $1 \mathrm{mV} / \mathrm{m}$ in the $X$ direction. Here, $e$ is the fundamental charge.

The $180^{\circ}$ phase difference between $|\boldsymbol{B}|$ and $n_{e}$ fluctuations and the thin current sheet in $X$ suggest an oscillating current sheet (surface wave) rather than a plane wave. To test this hypothesis, we generate a model of an oscillating current sheet and have a virtual spacecraft traverse the structure. The displacement is in the $X$ direction whereas the real part of the wave vector $(\boldsymbol{k})$ is in the $Y$ direction consistent with the $7.5 \mathrm{~Hz}$ oscillation and a $600 \mathrm{~km} / \mathrm{s}$ speed in the $-Y$ direction.

Our model uses GSE coordinates since finding an accurate $L-M-N$ coordinate system was difficult due to the strong fluctuations. As observed, the current sheet normal ( $N$ direction) is primarily in the $X$ direction, the largest change in $\boldsymbol{B}$ ( $L$ direction) is primarily in the $Z$ direction, and the current $(-M$ direction) is in the $Y$ direction, so a coordinate transformation is not necessary to test a simple model. Figure $3 a$ displays a cross section of the current sheet model as a cut in the $X$ direction. $J_{y}$ is set as $J_{0} \operatorname{sech}\left(x / x_{0}\right)$, with $J_{0}=1.4 \mu \mathrm{A} / \mathrm{m}^{2}$ (Figure $2 \mathrm{~g}$ ). $x_{0}$ linearly increases from $\sim 7 \mathrm{~km}$ to $\sim 18 \mathrm{~km}$ with increasing $x$ to best reproduce the shape of $B_{z}$ without accounting for fluctuations (Figure $2 \mathrm{~d}$ ). $B_{z}$ is calculated as $\int \mu_{\mathrm{o}} J_{y} \mathrm{~d} x$. The electron density $\left(n_{\mathrm{e}}\right)$ is derived from pressure balance using a steadily increasing sum of $T_{\mathrm{i}}$ and $T_{\mathrm{e}}$ as observed. Pressure balance (including ion ram pressure) results in a reasonable reproduction of the observed density (Figure $2 \mathrm{c}$ ). Since $V_{\mathrm{e}}$ (not shown) dominates the current, $E_{x}$ is derived as $J_{y} B_{z} / e n_{\mathrm{e}}$.

Figure $3 b$ shows the approximate path of MMS1 in the $X-Z$ plane and Figure $3 c$ shows the path in the $X-Y$ plane. Figure $3 c$ (not to scale) also depicts a corrugated current sheet in the $Y$ direction. The corrugation oscillates at $7.5 \mathrm{~Hz}$ (in agreement with the observed fluctuations) with a displacement of $\sim 4 \mathrm{~km}$ in the $X$ direction (best fit to fluctuations). The wavelength of the corrugation ( $Y$ direction) is $\sim 80 \mathrm{~km}$, consistent with the measured speed of the $\boldsymbol{B}$ fluctuations (Figures 20 and $2 \mathrm{p}$ ). A virtual spacecraft is started at $x=5 \mathrm{~km}$, then penetrates the oscillating current sheet with a speed of $-40 \mathrm{~km} / \mathrm{s}$. The speed of the virtual spacecraft, the wave phase, and the displacement of the current sheet $(4 \mathrm{~km})$ are optimized to reproduce the observed fluctuations plotted in Figures $3 \mathrm{~d}-3 \mathrm{~g}$. Figure $3 \mathrm{~d}$ shows $|\boldsymbol{B}|$ fluctuations predicted by the model (black line) due to the current sheet oscillations as the spacecraft traveled in the $-X$ direction. The red trace is the observed $|\boldsymbol{B}|$ from Figure $2 \mathrm{~d}$. The fluctuations come from a combination of the current sheet oscillation (displacement of 

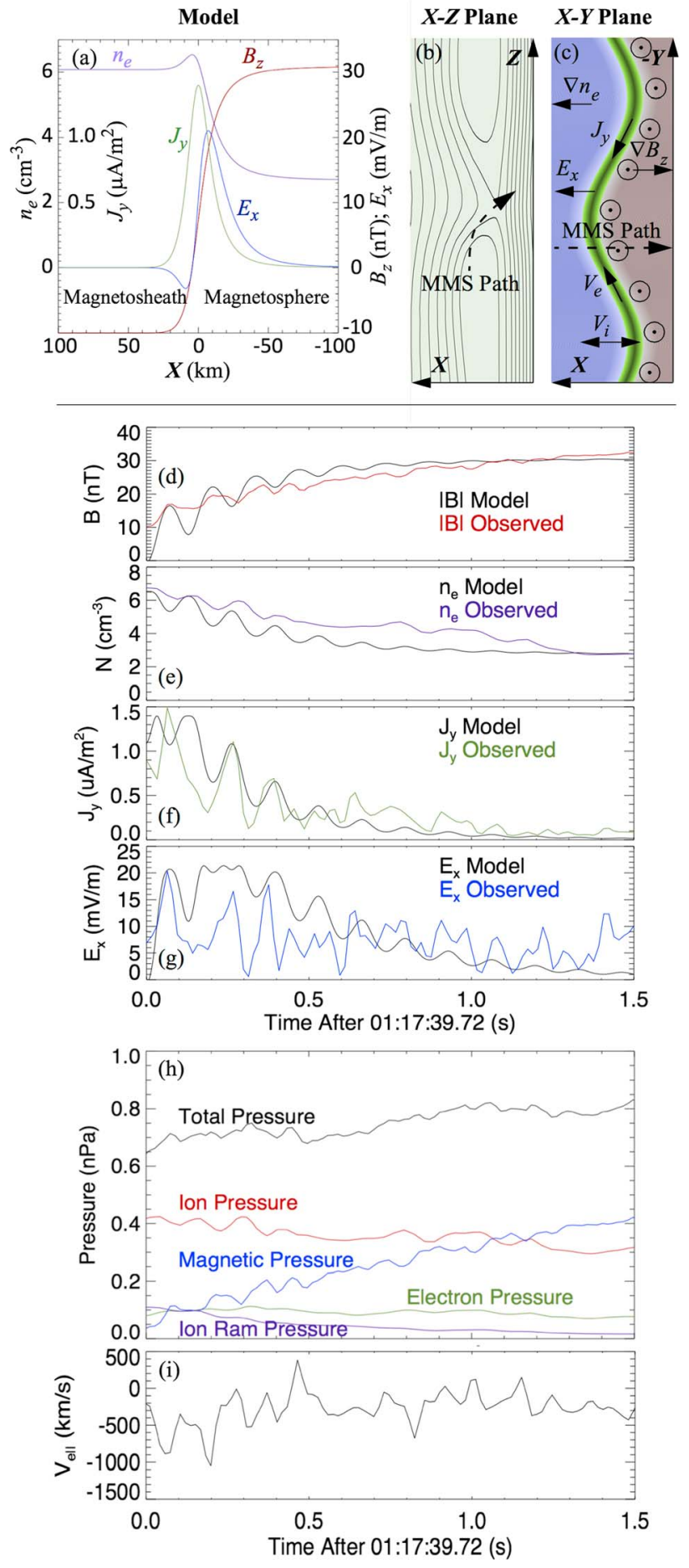

Figure 3. (a) A cross section of the model current sheet ignoring the fluctuations. $J_{y}$ (green) is modeled as a sech function (see text) to best reproduce $B_{Z}$ (red). $n_{\mathrm{e}}$ (purple) is calculated to maintain pressure balance with slowly rising $T_{\mathrm{i}}$ and $T_{\mathrm{e}}$. $E_{X}$ (blue) is $J_{y} B_{z} / n_{\mathrm{e}} e$. (b) The MMS path in $X-Z$ plane. (c) $\mathrm{A}$ cartoon of an oscillating corrugated current sheet. $J_{y}$ is in green. The highdensity region is in blue, and the strong $|\boldsymbol{B}|$ region is in brown. $(\mathrm{d}-\mathrm{g}) \mathrm{A}$ comparison of the predicted fluctuations by the model (black traces) with the observed $|\boldsymbol{B}|, n_{\mathrm{e}}, J_{y}$, and $E_{x}$. (h) The total pressure (black), $P_{\mathrm{i}}$ (red), $\boldsymbol{B}^{2} / 2 \mu_{\mathrm{o}}$ (blue), and $P_{\mathrm{e}}$ (green) from observations. The ion ram pressure (purple) is an estimate, $1 / 2 n_{\mathrm{e}} m_{\mathrm{i}} V_{\mathrm{ix}}{ }^{2}$, where $m_{\mathrm{i}}$ is the proton mass. (i) $V_{\mathrm{e} \|}$ as measured.
$4 \mathrm{~km}$ at $7.5 \mathrm{~Hz}$ ) and the positive slope of $|\boldsymbol{B}|$ (Figure 3a). The model predicts that the fluctuations should diminish as a spacecraft moves away from the current sheet.

Figure $3 e$ plots $n_{\mathrm{e}}$ fluctuations predicted by the model (black line) and the observed $n_{\mathrm{e}}$ fluctuations from Figure 2c (purple line). Again, the fluctuations come from a combination of the current sheet oscillation and the negative slope of $n_{\mathrm{e}}$ (Figure 3a). Importantly, the fluctuations in $|\boldsymbol{B}|$ and $n_{\mathrm{e}}$ are expected to be $\sim 180^{\circ}$ out of phase, which is in agreement with the observations. Figure $3 f$ displays the predicted $J_{y}$ fluctuations (green line) and the observations (black line). Figure $3 \mathrm{~g}$ displays $E_{X}$ in the same fashion. Both $J_{y}$ and $E_{x}$ are peaked rather than monotonic in $X$ (Figure $3 a$ ), so the oscillating current sheet has more complex results. As a spacecraft crosses a peak in $J_{y}$, the model predicts a double-peaked current as a function of time. On the negative slope in the $+X$ region (Figure $3 a$ ), the oscillations become more periodic in time. The $E_{X}$ peak is shifted in $X$ from the $J_{y}$ peak due to the change in $\boldsymbol{B}$, but a multiple peak in time is also predicted. The exact shape of the time-domain signal, however, is sensitive to the spatial shape of the peak in $J_{y}$ and $E_{x}$, so an exact agreement is not expected.

Figures $3 d-3 g$ reveal an excellent qualitative agreement and good quantitative agreement with the observed $|\boldsymbol{B}|, n_{\mathrm{e}}, J_{y}$, and $E_{x}$ signals. This agreement suggests a current sheet corrugation with displacement in the $X$ direction of $\sim 4 \mathrm{~km}$ and traveling in the $-Y$ direction, which is consistent with a type of electromagnetic drift wave. Figure $3 \mathrm{~h}$ verifies that the fluctuations in ion pressure and magnetic pressure are $180^{\circ}$ out of phase and that $P_{\mathrm{e}}$ is nearly constant across the current sheet. 
Ion motion must also be considered since the plasma must remain quasineutral on scales greater than the Debye length $(\sim 50 \mathrm{~m})$. The current sheet oscillations displace electrons $+4 \mathrm{~km}$ with an angular frequency $\omega=2 \pi 7.5 \mathrm{~Hz}$ in the $X$ direction, so the oscillating displacement speed $\left(V_{\mathrm{ex}}\right)$ peaks at roughly $+200 \mathrm{~km} / \mathrm{s}$ (also seen in electron data, Figure $2 \mathrm{f}$ ). This speed is far higher than can be produced by a simple ion inertial response. The current sheet oscillation frequency is much higher than $f_{\mathrm{ci}}$, so the ion inertial response is $\left|\delta V_{\mathrm{ix}}\right| \sim e\left|\delta E_{x}\right| / m_{\mathrm{i}} \omega$, where $\delta V_{\mathrm{ix}}$ is the $V_{\mathrm{ix}}$ fluctuation amplitude, $\delta E_{X}$ is the $E_{X}$ fluctuation amplitude, and $m_{\mathrm{i}}$ is the ion mass. $\left|\delta V_{\mathrm{ix}}\right|$ is at most $\sim 20 \mathrm{~km} / \mathrm{s}$ if $\delta E_{X}$ is $\sim \pm 10 \mathrm{mV} / \mathrm{m}$.

The ion motion, however, may be more complex than a simple inertial response. $E_{x}$ near the EDR slows and deflects the incoming ions. $V_{i x}$ is $\sim-80 \mathrm{~km} / \mathrm{s}$ in the magnetosheath (Figure 2e, 01:17:39 UT) and changes to $\sim 20 \mathrm{~km} / \mathrm{s}$ on the magnetosphere side of the current sheet (Figure 2e, 01:17:41 UT), a change of $\sim 100 \mathrm{~km} / \mathrm{s}$. Furthermore, our model and observations indicate that the current sheet is moving $\sim 40 \mathrm{~km} / \mathrm{s}$ in the $+X$ direction. Since $E_{X}$ carries $\sim 500 \mathrm{~V}$ in net potential across the current sheet, it can reflect or divert the incoming ions. If so, the point at which the ion inflow is slowed and diverted can oscillate, causing the density to fluctuate, at least on the magnetosheath side of the current sheet. A full kinetic analysis is required to determine the ion density response in the drift wave.

Figure 3i, which plots $\boldsymbol{V}_{\mathrm{e}} \cdot \boldsymbol{B} /|\boldsymbol{B}|$, indicates significant electron motion along $\boldsymbol{B}$. These data suggest that ion response alone does not maintain quasineutrality and that the corrugated drift wave has variation along $\boldsymbol{B}$. Strong $V_{\mathrm{ez}}$ motion is associated with intense $E_{\|}$at high frequencies [Newman et al., 2001; Roux et al., 2011; Stawarz et al., 2015]. Since the bursts of $E_{\|}$have short durations (tens of milliseconds), it is possible that the observations in Figure $3 \mathrm{i}$ are not representative since they are averaged over $30 \mathrm{~ms}$. Thin (a few km) current sheets or rapidly fluctuating currents $(\sim 10 \mathrm{~ms})$ with $\left|\delta V_{\mathrm{ez}}\right| \sim 5000 \mathrm{~km} / \mathrm{s}$ would be Buneman unstable and produce the observed nonlinear $E_{\|}$structures [e.g., Newman et al., 2001]. Alternatively, lesser $\left|\delta V_{\mathrm{ez}}\right|$ speeds could produce a myriad of oblique instabilities [Kindel and Kennel, 1971; Stawarz et al., 2015]. The narrow spikes in the $E_{\|}$emissions near 01:17:40 UT are consistent with intense parallel currents, which either are in thin sheets or have short durations.

\section{Discussion and Conclusions}

In five of six confirmed EDR regions, $\boldsymbol{B}$ fluctuations, some appearing turbulent, are seen in the magnetopause current sheet with frequencies higher than $f_{\mathrm{ci}}$ but lower than $f_{\mathrm{lh}}$. In the sixth event, the MMS spacecraft did not fully cross the magnetopause current sheet, so fluctuations could not have been detected. Bursts of high-frequency $E_{\|}$are concurrent with the fluctuations in $\boldsymbol{B}$. Fluctuations in $\boldsymbol{B}, n_{\mathrm{e}}, \boldsymbol{J}$, and $\boldsymbol{E}$ are consistent with a corrugated current sheet with the real part of $\boldsymbol{k}$ primarily in the direction along the $X$ line of the magnetic reconnection ( $Y$ direction). The displacement is normal to the current sheet ( $X$ direction), so the waves are transverse. Importantly, $\boldsymbol{k}$ also has a finite component along $\boldsymbol{B}$ ( $Z$ direction). The localization in the current sheet normal direction ( $X$ direction) indicates that $\boldsymbol{k}$ has a significant imaginary component in $X$, such as in a surface wave. The electromagnetic drift wave and turbulence are mostly from electron motion. Ion participation requires kinetic modeling or simulation.

The energy source of these electromagnetic drift waves is unlikely due to $\nabla P_{\mathrm{e}}$ since $P_{\mathrm{e}}$ is nearly constant across the current sheet (Figure 3h). Pressure balance requires either ion thermal pressure or ram pressure, the latter of which causes the strong Hall electric field $\left(E_{x}\right)$. Indirectly, a possible source of the instability is a two-stream instability from the strong electron velocity $\left(V_{\text {ey }}\right)$ driven by the Hall electric field $\left(E_{x}\right)$. A strong $E_{x}$ (order of $10 \mathrm{mV} / \mathrm{m}$ ) is common to all of the asymmetric EDR encounters that we examined [Burch et al., 2016b; Burch and Phan, 2016; Phan et al., 2016; Chen et al., 2016] and seen in simulations [Cassak and Shay, 2007; Hesse et al., 2014, 2016].

Similar waves have been seen in 3D simulations [Daughton, 2003; Pritchett et al., 2012; Roytershteyn et al., 2013; Price et al., 2016] and in laboratory experiments [Ji et al., 2004, 2005, 2008]. The observed waves have many of the properties of the electromagnetic drift waves discussed by Ji et al. [2004, 2005, 2008], who investigated an obliquely propagating electromagnetic wave in the lower hybrid frequency range driven by the relative drifts between electrons and ions. The electromagnetic drift waves observed in the laboratory were reproduced with smaller amplitudes in simulations [Roytershteyn et al., 2013]. The wavelength seems to be in agreement in that $k_{y} \sqrt{\rho_{\mathrm{e}} \rho_{\mathrm{i}}} \sim 1$ [Daughton, 2003; Roytershteyn et al., 2013]. 
The LDHI has been suggested as a possible source of observed waves near the lower hybrid frequency in the vicinity magnetopause reconnection regions [e.g., Bale et al., 2002; Eastwood et al., 2009; Zhou et al., 2009]. However, in its simplest form, the LHDI [e.g., Davidson and Gladd, 1975; Davidson et al., 1977]; Yoon et al., 2008] is expected (1) to produce fluctuations at $f_{\mathrm{lh}}$, (2) to be electrostatic (longitudinal), (3) to have $k_{\|}=0$, and (4) to draw its energy from an electron drift due to $\nabla P_{\mathrm{e}}$. The LHDI has been extended to include waves below $f_{\mathrm{lh}}$ [e.g., Bale et al., 2002] and/or have an electromagnetic component [Daughton, 2003]. However, the observed drift waves and turbulence do not satisfy $k_{\|}=0$, and $\nabla P_{\mathrm{e}}$ appears not to play a role. As such, we cannot unequivocally assign the source of the reported electromagnetic drift waves to the LHDI. The low-frequency fluctuations are accompanied by strong, higher-frequency bursts of $E_{\|}$[Ergun et al., 2016b] and by parallel electron flows, which indicate a finite $k_{\|}$. We hypothesize that, at large enough amplitudes (displacement in the $X$ direction), ion motion is unable to maintain quasineutrality indicating that electron motion along $\boldsymbol{B}$ is required. The electron motion along $\boldsymbol{B}$ may be confined to thin, intense $J_{\|}$or short-duration, intense $J_{\|}$exciting the parallel electric fields [Newman et al., 2001; Roux et al., 2011; Stawarz et al., 2015].

Even with such few examples, it appears that the current sheet near the EDR of asymmetric reconnection may be unstable and that the $\boldsymbol{B}$ fluctuations are linked to the $E_{\|}$fluctuations. We note that the apparent link is confounded by the possible presence of cold plasma, which can stimulate bursts of $E_{\|}$as well [Ergun et al., 2016b]. Interestingly, two examples of symmetric reconnection [Øieroset et al., 2016; Eriksson et al., 2016] do not show $B$ fluctuations.

We can conclude that the current sheet near the asymmetric magnetic reconnection region appears to be unstable to a type of low-frequency electromagnetic drift wave, which can generate turbulence [Price et al., 2016] and are associated with bursts of strong $E_{\|}$. It is unclear, but possible, that the low-frequency turbulence can influence the large-scale structure of magnetic reconnection generating a time-dependent, patchy reconnection process.

\section{Acknowledgments}

This work was funded by the NASA MMS project. The authors recognize the tremendous effort in developing and operating the MMS spacecraft and instruments and sincerely thank all involved. The authors thank Bill Daughton for useful discussions. MMS data are open to the public.

\section{References}

Bale, S. D., F. S. Mozer, and T. Phan (2002), Observation of lower hybrid drift instability in the diffusion region at a reconnecting magnetosphere, Geophys. Res. Lett., 29(24), 2180, doi:10.1029/2002GL016113.

Birn, J., et al. (2001), Geospace Environmental Modeling (GEM) magnetic reconnection challenge, J. Geophys. Res., 106, 3715-3719, doi:10.1029/1999JA900449.

Burch, J. L., and J. F. Drake (2009), Reconnecting magnetic fields, Am. Sci., 97, 392-399.

Burch, J. L., and T. D. Phan (2016), Magnetic reconnection at the dayside magnetopause: Advances with MMS, Geophys. Res. Lett., 43, 8327-8338, doi:10.1002/2016GL069787.

Burch, J. L., T. E. Moore, R. B. Torbert, and B. L. Giles (2016a), Magnetospheric multiscale overview and science objectives, Space Sci. Rev., 199, 5-21, doi:10.1007/s11214-015-0164-9.

Burch, J. L., et al. (2016b), Electron-scale measurements of magnetic reconnection in space, Science, doi:10.1126/science.aaf2939, in press

Cassak, P. A., and M. A. Shay (2007), Scaling of asymmetric magnetic reconnection: General theory and collisional simulations, Phys. Plasmas, 14, 102114, doi:10.1063/1.2795630.

Chen, F. F. (1984), Plasma Physics and Controlled Fusion, pp. 218-223, Plenum Press, New York.

Chen, L.-J., et al. (2016), Electron energization and mixing observed by MMS in the vicinity of an electron diffusion region during magnetopause reconnection, Geophys. Res. Lett., 43, 6036-6043, doi:10.1002/2016GL069215.

Daughton, W. (2003), Electromagnetic properties of the lower-hybrid drift instability in a thin current sheet, Phys. Plasmas, 10, 3103-3119.

Davidson, R. C., and N. T. Gladd (1975), Anomalous transport properties associated with the lower-hybrid-drift instability, Phys. Fluids, 18, 1327-1335.

Davidson, R., N. Gladd, C. Wu, and J. Huba (1977), Effects of finite plasma beta on the lower-hybrid drift instability, Phys. Fluids, $20,301$.

Drake, J. F., M. Swisdak, H. Che, and M. A. Shay (2006), Electron acceleration from contracting magnetic islands during reconnection, Nature, 443, 553-556, doi:10.1038/nature05116.

Eastwood, J. P., T. D. Phan, S. D. Bale, and A. Tjulin (2009), Observations of turbulence generated by magnetic reconnection, Phys. Rev. Lett., 102, 035001.

Egedal, J., W. Daughton, A. Le, and A. L. Borg (2015), Double layer electric fields aiding the production of energetic flat-top distributions and superthermal electrons within the exhausts from magnetic reconnection, Phys. Plasmas, 22(10), 101208.

Ergun, R. E., et al. (2016a), Magnetospheric multiscale satellites observations of parallel electric fields associated with magnetic reconnection, Phys. Rev. Lett., 116, 235102, doi:10.1103/PhysRevLett.116.235102.

Ergun, R. E., et al. (2016b), Magnetospheric Multiscale observations of large-amplitude, parallel, electrostatic waves associated with magnetic reconnection at the magnetopause, Geophys. Res. Lett., 43, 5626-5634, doi:10.1002/2016GL068992.

Ergun, R. E., et al. (2016c), The axial double probe and fields signal processing for the MMS mission, Space Sci. Rev., 199, 67-188, doi:10.1007/ s11214-014-0115-x.

Eriksson, S., et al. (2016), Magnetospheric multiscale observations of the electron diffusion region of large guide field magnetic reconnection, Phys. Rev. Lett., 117, 015001, doi:10.1103/PhysRevLett.117.015001.

Hesse, M., and D. Winske (1998), Electron dissipation in collisionless magnetic reconnection, J. Geophys. Res., 103, 26,479-26,486, doi:10.1029/ 98JA01570. 
Hesse, M., N. Aunai, D. Sibeck, and J. Birn (2014), On the electron diffusion region in planar, asymmetric, systems, Geophys. Res. Lett., 41, 8673-8680, doi:10.1002/2014GL061586.

Hesse, M., Y.-H. Liu, L.-J. Chen, N. Bessho, M. Kuznetsova, J. Birn, and J. L. Burch (2016), On the electron diffusion region in asymmetric reconnection with a guide magnetic field, Geophys. Res. Lett., 43, 2359-2364, doi:10.1002/2016GL068373.

Ji, H., S. Terry, M. Yamada, R. Kulsrud, A. Kuritsyn, and Y. Ren (2004), Electromagnetic fluctuations during fast reconnection in a laboratory plasma, Phys. Rev. Lett., 92, 115001.

Ji, H., R. Kulsrud, W. Fox, and M. Yamada (2005), An obliquely propagating electromagnetic drift instability in the lower hybrid frequency range, J. Geophys. Res., 110, A08212, doi:10.1029/2005JA011188.

Ji, H., Y. Ren, M. Yamada, S. Dorfman, W. Daughton, and S. P. Gerhardt (2008), New insights into dissipation in the electron layer during magnetic reconnection, Geophys. Res. Lett., 35, L13106, doi:10.1029/2008GL034538.

Kindel, J. . M., and C. . F. Kennel (1971), Topside current instabilities, J. Geophys. Res., 76, 3055-3078, doi:10.1029/JA076i013p03055.

Krall, N. A., and P. C. Liewer (1971), Low frequency instabilities in magnetic pulses, Phys. Rev. A, 4, 2094-2103.

Lapenta, G., D. Krauss-Varban, H. Karimabadi, J. D. Huba, L. I. Rudakov, and P. Ricci (2006), Kinetic simulations of x-line expansion in 3D reconnection, Geophys. Res. Lett., 33, L10102, doi:10.1029/2005GL025124.

Lapenta, G., S. Markidis, M. V. Goldman, and D. L. Newman (2015), Secondary reconnection sites in reconnection-generated flux ropes and reconnection fronts, Nat. Phys., 11, 690-695, doi:10.1038/nphys3406.

Le Contel, O., et al. (2016), The search-coil magnetometer for MMS, Space Sci. Rev., 199, 257-282, doi:10.1007/s11214-014-0096-9.

Li, T. C., J. F. Drake, and M. Swisdak (2012), Supression of energetic electron transport in flares by double layers, Astrophys. J., 757, doi:10.1088/ 0004-637X/757/1/20

Lindqvist, P.-A., et al. (2016), The spin-plane double probe instrument for MMS, Space Sci. Rev., 199, 137-165, doi:10.1007/s11214-014-0116-9.

McBride, J. B., E. Ott, J. P. Boris, and J. H. Orens (1972), Theory and simulation of turbulent heating by the modified two-stream instability, Phys. Fluids, 15, 2367.

McFadden, J., C. Carlson, D. Larson, J. Bonnell, F. Mozer, V. Angelopoulos, K.-H. Glassmeier, and U. Auster (2008), Structure of plasmaspheric plumes and their participation in magnetopause reconnection: First results from THEMIS, Geophys. Res. Lett., 35, L17S10, doi:10.1029/ 2008 GL033677.

Newman, D. L., M. V. Goldman, R. E. Ergun, and A. Mangeney (2001), Formation of double layers and electron holes in a current-driven space plasma, Phys. Rev. Lett., 87, 255001, doi:10.1103/PhysRevLett.87.255001.

Øieroset, M., et al. (2016), MMS observations of large guide field symmetric reconnection between colliding reconnection jets at the center of a magnetic flux rope at the magnetopause, Geophys. Res. Lett., 43, 5536-5544, doi:10.1002/2016GL069166.

Paschmann, G., M. Øieroset, and T. Phan (2013), In-situ observations of reconnection in space, Space Sci. Rev., 47, 309-341, doi:10.1007/ 978-1-4899-7413-6_12.

Phan, T. D., et al. (2016), MMS observations of electron-scale filamentary currents in the reconnection exhaust and near the $\mathrm{X}$ line, Geophys. Res. Lett., 43, 6060-6069, doi:10.1002/2016GL069212.

Pollock, C., et al. (2016), Fast plasma investigation for magnetospheric multiscale, Space Sci. Rev., 199, 331-406, doi:10.1007/ s11214-016-0245-4.

Price, L., M. Swisdak, J. F. Drake, P. A. Cassak, J. T. Dahlin, and R. E. Ergun (2016), The effects of turbulence on three-dimensional magnetic reconnection at the magnetopause, Geophys. Res. Lett., 43, 6020-6027, doi:10.1002/2016GL069578.

Pritchett, P. L., F. S. Mozer, and M. Wilber (2012), Intense perpendicular electric fields associated with three-dimensional magnetic reconnection at the subsolar magnetopause, J. Geophys. Res., 117, A06212, doi:10.1029/2012JA017533.

Roux, A., P. Robert, O. Le Contel, V. Angelopoulos, U. Auster, J. Bonnell, C. M. Cully, R. E. Ergun, and J. P. McFadden (2011), A mechanism for heating electrons in the magnetopause current layer and adjacent regions, Ann. Geophys., 29, 2305-2316, doi:10.5194/ angeo-29-2305-2011.

Roytershteyn, V., S. Dorfman, W. Daughton, H. Ji, and M. Yamada (2013), Electromagnetic instability of thin reconnection layers: Comparison of three-dimensional simulations with MRX observations, Phys. Plasmas, 20, 061212, doi:10.1063/1.4811371.

Russell, C. T., et al. (2016), The magnetospheric multiscale magnetometers, Space Sci. Rev., 199, 189-256, doi:10.1007/s11214-014-0057-3.

Shay, M. A., J. F. Drake, R. E. Denton, and D. Biskamp (1998), Structure of the dissipation region during collisionless magnetic reconnection, J. Geophys. Res., 103, 9165-9176, doi:10.1029/97JA03528.

Stawarz, J. E., R. E. Ergun, and K. A. Goodrich (2015), Generation of high-frequency electric field activity by turbulence in the Earth's magnetotail, J. Geophys. Res. Space Physics, 120, 1845-1866, doi:10.1002/2014JA020166.

Su, Y.-J., J. E. Borovsky, M. F. Thomsen, R. C. Elphic, and D. J. McComas (2000), Plasmaspheric material at the reconnecting magnetopause, J. Geophys. Res., 105, 7591-7600, doi:10.1029/1999JA000266.

Torbert, R. B., et al. (2016), The FIELDS instrument suite on MMS: Scientific objectives, measurements, and data products, Space Sci. Rev., 199, 105-135, doi:10.1007/s11214-014-0109-8.

Wang, S., et al. (2016), Two-scale ion meandering caused by the polarization electric field during asymmetric reconnection, Geophys. Res. Lett., 43, 7831-7839, doi:10.1002/2016GL069842.

Yoon, P. H., Y. Lin, X. Y. Wang, and A. T. Y. Lui (2008), Theory and simulation of lower-hybrid drift instability for current sheet with guide field, Phys. Plasmas, 15(11), 1121037, doi:10.1063/1.3013451.

Zhou, M., et al. (2009), Observation of waves near lower hybrid frequency in the reconnection region with thin current sheet, J. Geophys. Res., 114, A02216, doi:10.1029/2008JA013427. 